\title{
An efficient immunodetection method for histone modifications in plants
}

\author{
Geovanny Nic-Can', Sara Hernández-Castellano², Angela Kú-González ${ }^{1}$, Víctor M Loyola-Vargas ${ }^{1}$ and Clelia De-la-Peña ${ }^{2 *}$
}

\begin{abstract}
Background: Epigenetic mechanisms can be highly dynamic, but the cross-talk among them and with the genome is still poorly understood. Many of these mechanisms work at different places in the cell and at different times of organism development. Covalent histone modifications are one of the most complex and studied epigenetic mechanisms involved in cellular reprogramming and development in plants. Therefore, the knowledge of the spatial distribution of histone methylation in different tissues is important to understand their behavior on specific cells.

Results: Based on the importance of epigenetic marks for biology, we present a simplified, inexpensive and efficient protocol for in situ immunolocalization on different tissues such as flowers, buds, callus, somatic embryo and meristematic tissue from several plants of agronomical and biological importance. Here, we fully describe all the steps to perform the localization of histone modifications. Using this method, we were able to visualize the distribution of H3K4me3 and H3K9me2 without loss of histological integrity of tissues from several plants, including Agave tequilana, Capsicum chinense, Coffea canephora and Cedrela odorata, as well as Arabidopsis thaliana.
\end{abstract}

Conclusions: There are many protocols to study chromatin modifications; however, most of them are expensive, difficult and require sophisticated equipment. Here, we provide an efficient protocol for in situ localization of histone methylation that dispenses with the use of expensive and sensitive enzymes. The present method can be used to investigate the cellular distribution and localization of a wide array of proteins, which could help to clarify the biological role that they play at specific times and places in different tissues of various plant species.

Keywords: Agave tequilana, Arabidopsis thaliana, Capsicum chinense, Cedrela odorata, Coffea canephora, Epigenetic mechanism, Histone H3, H3K4me3, H3K9me2, Immunodetection

\section{Background}

In eukaryotes, including higher plants, the information deposited in the DNA is found compacted into chromatin. However, additional layers of information, known as epigenetics, are imposed on DNA. Furthermore, different molecules can modify the chromatin structure in many ways. For instance, in plants, DNA can be methylated in the deoxycytocine residues in the context of all sequences, from symmetric CG and CHG (where $\mathrm{H}=\mathrm{A}$, $\mathrm{T}$ or $\mathrm{C}$ ) to asymmetric $\mathrm{CHH}$ [1], whereas histones that conform to the nucleosome (H2A, H2B, H3 and $\mathrm{H} 4$ ) can be covalently modified in their $\mathrm{N}$-terminal tails by methylation, acetylation and phosphorylation, among

\footnotetext{
* Correspondence: clelia@cicy.mx

¿ Unidad de Biotecnología, Centro de Investigación Científica de Yucatán, Calle 43 No. 130, Col. Chuburná de Hidalgo, Mérida CP 97200, Yucatán, México

Full list of author information is available at the end of the article
}

others [2]. All these modifications are dynamic and reversible and, ultimately, play an essential role in the transcriptional regulation of genes $[3,4]$.

Recent plant genome-wide analysis, particularly in Arabidopsis thaliana, rice and maize, has been used to increase knowledge of the plant epigenome and its response to environmental stimuli or developmental cues [5-7]. These studies begin to reveal the correlation between epigenetic marks and transcriptional activity. For instance, it has been shown that the trimethylation of histone $\mathrm{H} 3$ at lysine 4 (H3K4me3) is present, most of the time, at the promoter region of genes $[5,7,8]$, while that of the di- and trimethylation of histone $\mathrm{H} 3$ at lysine 36 (H3K36me2 and H3K36me3) are distributed over the transcribed region $[9,10]$, and both are abundant in highly expressed genes. By contrast, H3K9me2, a mark related to the formation of heterochromatin, has been found in both the promoter and the gene body $[11,12]$,

\section{Biomed Central}


whereas H3K27me3 is localized within the gene body $[13,14]$. Both marks (H3K9me2 and H3K27me3) have been associated with the repression of transcription [15].

Several protocols are available to study histones and their modifications, but the majority of them have been done and adapted to the model plant $A$. thaliana $[3,16,17]$. In addition, other techniques have also been used to examine the spatial distribution of histones and their covalent modifications in the cell, such as fluorescent in situ hybridization (FISH) [18] and immunolocalization [19-21]. Although these methods have enabled the visualization of histones' changes inside the cell, most of the time these methods are realized through complex techniques. These methods may also require sophisticated equipment, such as cryostat to section plant tissue. Another difficulty in these methods is the use of protease inhibitors and several enzymes to degrade the cell wall; these enzymes are typically expensive and sensitive to degradation. Furthermore, the use of squashing during immunolocalization preparation affects the interpretation of the results because the squashing or protoplasting may alter the cellular structures [22,23]. Although these mentioned methodologies have been used in many reports, they do not work well for all plant tissues, as other authors have found.

All these technical issues motivated us to find a simple, suitable and inexpensive protocol to detect in situ cellular distribution of histone modifications in a wide array of plants, tissues and conditions (in vitro and ex vitro). In the present work, we describe step-by-step the required instructions to carry out immunolocalization from paraffin-embedded tissue sections, focusing on several species of agronomical interest such as Agave tequilana, Capsicum chinense, Coffea canephora and Cedrela odorata, as well as the model plant A. thaliana. These plants were selected to test our protocol because of their economical and agronomical relevance (A. tequilana, C. canephora, C. chinense and C. odorata) and their molecular importance as a model (A. thaliana). Furthermore, the different behaviors of epigenetic modifications found in these plants could give us a clue about the molecular mechanism that influences their development.

\section{Results and discussion} Immunodetection procedure

In order to provide a suitable and standard methodology for in situ immunolocalization from FAA-fixed and paraffin-embedded plant tissues, we developed a simple and reproducible procedure for in situ immunolocalization in several tissues and plants, improving some of the steps reported by other protocols [23-27]. For instance, the use of only one step during the protein-antibody interaction has increased the antibodies' efficiency in several tissues in different plant growth conditions. Also, the time of fixation in different kinds of tissues was homogenous in all samples, even for those plants with rigid cell walls and particularly for timber species such as coffee and cedar. In addition, we have discarded the use of enzymes responsible for degrading the cell wall, and the use of dimethylsulfoxide, NP40 and other reagents frequently used to achieve the cellular permeabilization $[22,23,28]$, which increase the cost of the immunolocalization protocols. Instead of all the previously listed chemicals, we used a single step for the recovery of antigen sites without affecting the histological integrity, obtaining a clear-cut protein distribution.

To test the reproducibility of our method, several tissues of different plant species of economical and agronomical interest, such as Agave, chili pepper, coffee, cedar and Arabidopsis were collected and treated under the procedures summarized in Figure 1. Tissues of several plant species from both ex vitro and in vitro conditions (Figure 2) were fixed in formaldehyde. In most of the protocols, the fixation step must be optimized according to the type of plant or tissue $[23,28]$. In the protocol described here, the sample fixation was carried out with final concentration of 3.7\% formaldehyde, providing good results. In addition, the formaldehyde promotes a strong preservation of the cellular and chromosomal structure. After the fixation step, the samples were dehydrated and paraffin-embedded in order to obtain a solid sample that would maintain tissue integrity during the sectioning step. Once the sliced sections are obtained, the tissue can be used for subsequent probes or stored at $4^{\circ} \mathrm{C}$ for several months without loss of integrity.

In order to remove the paraffin from the tissue, the tissue sections were heated at $65^{\circ} \mathrm{C}$ and rinsed with xylene and then with ultraclear. These steps are needed to allow full elimination of the paraffin residues; otherwise, this substance presents one of principal interferences in the immunodetection process. After total paraffin removal, the tissue samples were rehydrated and the antigen retrieval was performed. This step represents a critical point, because it enables the antibody to access the protein antigens, thus increasing signal intensity during immunodetection [29].

By nature, some protein epitopes are hidden within the complex structure of proteins, making it difficult to visualize the results during the immunolocalization. In several protocols of in situ hybridization or immunodetection in plants, the use of proteases or enzymes to degrade the cell wall is necessary in order to increase the permeability of the cell and its contents $[23,28,30]$. However, these techniques sometimes fail to achieve an appropriate immunostaining for many proteins. A suitable alternative for the development of antigen retrieval without the use of enzymes, which has been applied for clinical proposes in humans [29], is through boiling 


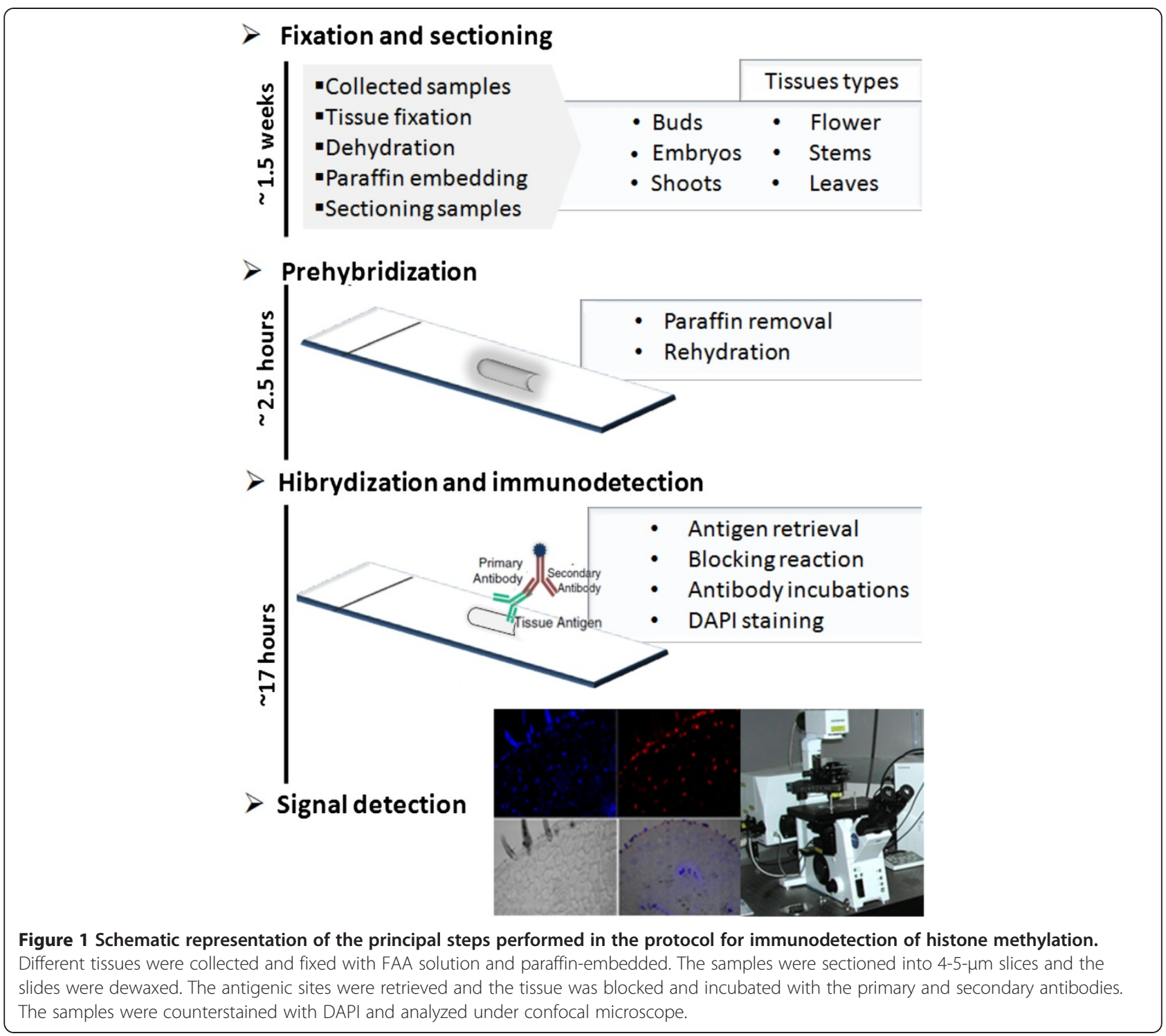

paraffin-embedded sections in the proper buffer. This procedure has the advantage of unmasking the tissue antigens, improving signal detection. Therefore, we investigated whether this procedure can be used in plants. We found that the heat-induced antigen retrieval using microwaved citrate buffer can indeed be applied to plant tissue without loss of histological integrity or protein detection. This step did not affect the tissue integrity in any of the plant species used here (Figure 3; transmitted light) and this step seems to be essential prior to the detection phase. Several protocols indicate that microwave irradiation enhances antigen retrieval, and it has been shown that this procedure is superior to traditional immunoflourescence in mammals [31,32]. Microwave irradiation has been used in plant tissues [33-35], and the use of this procedure improves the fixation process at low concentrations of formaldehyde. It is known that during heating, the energy provided helps to break some of the bonds formed during the fixation, increasing the number of available antigens in the cells and thus improving signal intensity $[24,36]$. On the other hand, some reports have proposed that the use of methanol/ acetone solution can preserve cellular architecture, allowing better access of the antibody to the antigen $[22,23]$. We tested this option; however, we did not find satisfactory results in most of the plants used (data not shown). Therefore, the antigen retrieval from the microwaved citrate buffer was chosen as a critical and essential step for immunodetection in all species studied here. We have found that the use of citrate solution with the microwave heating treatment increases the immunoreactivity, since the tissues without microwave-citrate treatment showed a substantial decrease or absence of signal (Additional files 1, 2 and 3). 

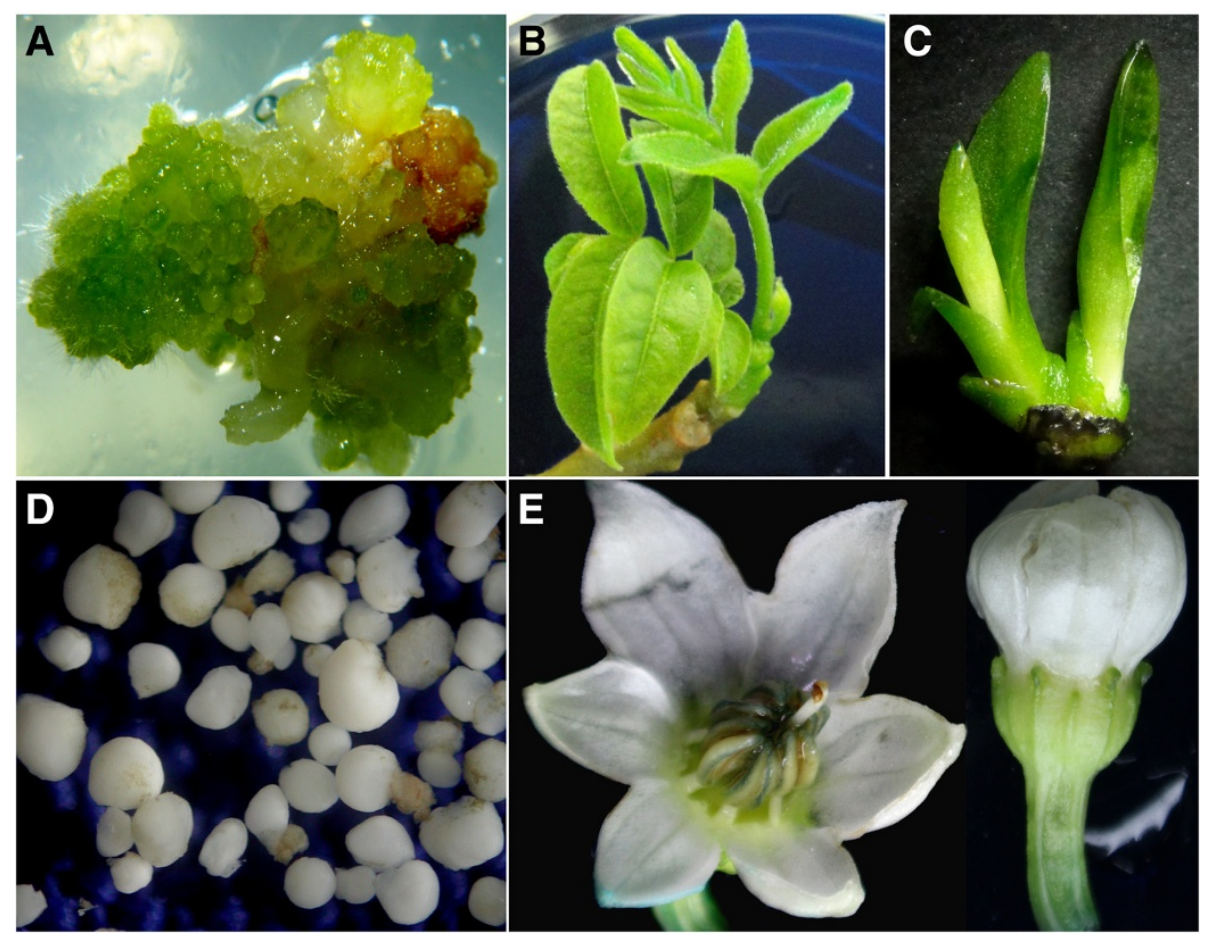

Figure 2 Plant material collected for the immunodetection proteins. (A) Callus of Arabidopsis thaliana. (B) Shoots and buds of Cedrela odorata. (C) Plantlets of Agave tequilana. (D) Somatic embryos of Coffea canephora obtained 56 days after induction. (E) Flowers of Capsicum chinense. A, C and $\mathbf{D}$ were obtained through cultures in vitro. $\mathbf{B}$ and $\mathbf{E}$ were collected from the field.

Another key factor that directly impacts the immunodetection is the quality of the primary antibodies, because good results obtained by Western blot do not always provide positive results for immunodetection. Therefore, selected monoclonal or polyclonal primary antibody dilutions need to be tested. We obtained satisfactory results using both monoclonal and polyclonal antibodies at a 1:200 dilution, a process which has been tested previously by Western blot and chromatin immunoprecipitation with different plant species $[37,38]$. On the other hand, the fluorescence-labeled secondary antibody is also important for the final signal detection, but this type of antibody needs to be chosen according to the microscope filter set and the working dilution should be determined experimentally. Here, Alexa Fluor ${ }^{\circ} 647$ at a 1:100 dilution provided good results. It must be noted that once the tissue sections are incubated with the fluorescence-labeled secondary antibody, they should be protected from light during all washes, as well as during DAPI staining and before to confocal analysis. In all steps, it is also important to prevent the tissue from drying, in order to decrease background, false signals or artifacts.

\section{Versatility of the immunodetection method in several plants, tissues and conditions}

According to the efficient and reproducible method described above, the results obtained by this protocol show that different plant species (Agave, chili pepper, cedar, coffee and Arabidopsis) can be treated with the same methodological conditions without loss of histological integrity, allowing a good recovery of antigenic sites and culminating with a better signal resolution. We collected material of both ex vitro and in vitro plant tissues, such as A. thaliana callus (in vitro) (Figure 2A), cedar buds (ex vitro) (Figure 2B), Agave plantlet stems (in vitro) (Figure $2 \mathrm{C}$ ), coffee somatic embryos (in vitro) (Figure 2D) and chili pepper flowers (ex vitro) (Figure 2E), and, in all cases, the reproducibility of our protocol for histone methylation localization was noted.

It was also observed that the signal intensity in all tissues of histone H3 and DAPI was well defined. Furthermore, a clean and clear signal of histone $\mathrm{H} 3$ between the cell localization of histone $\mathrm{H} 3$ and its localization inside the nucleus was observed (Figures 3). To discard the effect of autofluorescence, tissue sections were exposed without primary antibodies, which served as the negative control for both tissue sections as well as secondary antibody nonspecificity (data not shown). One of the most studied histone marks in plants and animals is H3K4me3, which is related to gene expression and, therefore, very dynamic $[7,8]$. In contrast, H3K9me2 represents a gene-silencing mark that promotes heterochromatin formation [39].

To test our protocol, we observed the distribution of antagonistic marks (H3K4me3 and H3K9me2) in several 


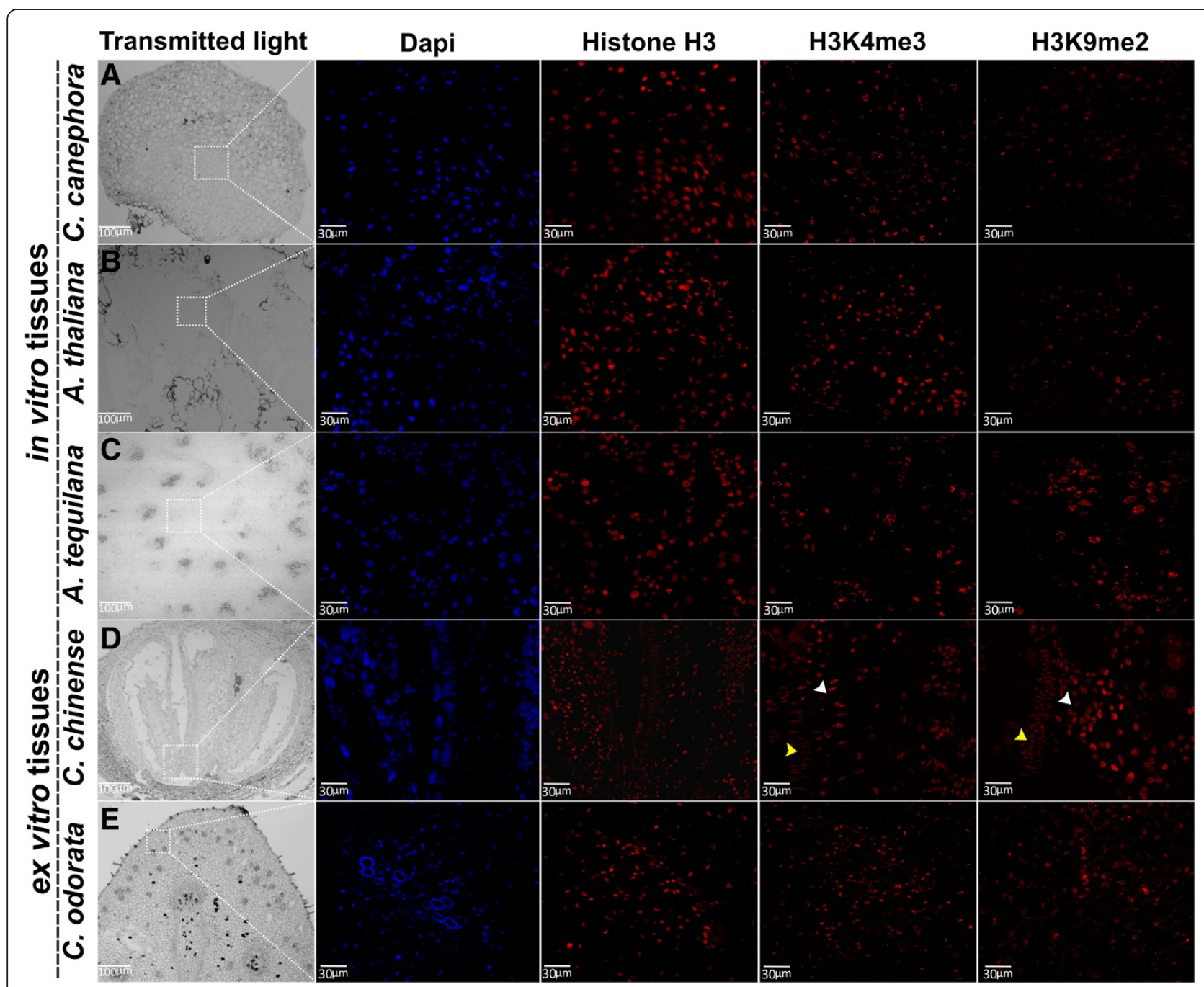

Figure 3 Immunodetection of histone modifications in tissues of several plant species. Immunodetection was performed using antibodies against H3K4me3 and H3K9me2, using the histone $\mathrm{H} 3$ as positive control. Panels from left to right show the confocal images of transmitted light, DAPI, histone H3, H3K4me3 and H3K9me2. Dashed squares represent the close-up of the sites detected by immunoflourescence with the antibodies mentioned above in the globular embryo of Coffea canephora (A), in meristematic zones in the callus of Arabidopsis thaliana (B), in the shoot apex of Agave tequilana (C), in the endothecium (yellow arrowhead) and in the lobes cells of anthers (white arrowhead) in Capsicum chinense (D) and in the proximal cells to epidermis of Cedrela odorata (E).

plant tissues and conditions (Figures 3 and 4). It was found that both epigenetic marks were highly dynamic, and these presented different subcellular distributions depending on the tissue of each plant species. For instance, in the somatic embryo at the globular stage of $C$. canephora, the results revealed that spatial distribution of H3K4me3 was significantly different in comparison with $\mathrm{H} 3 \mathrm{~K} 9 \mathrm{me} 2$, which presented a slight decrease in its signal in the center of this tissue, the region where the procambium cells would establish (Figure 3A). In the callus of $A$. thaliana, it was observed that the presence of H3K4me3 was prominent in those cells located at the meristematic zones (Figure 3B), while the H3K9me2 had lower signal. In the case of the transversal cut of $A$. tequilana plants, the highest levels of H3K9me2 were detected in cells of the shoot apex in comparison to H3K4me3 (Figure 3C).

In the $C$. chinense flower, a longitudinal cut revealed a low signal of H3K4me3 in the endothecium cells, as well as in the cells of the central lobes of the anthers. However, the levels of $\mathrm{H} 3 \mathrm{~K} 9 \mathrm{me} 2$ in the same cells were higher than H3K4me3 (Figure 3D). In the vegetative bud of C. odorata, specifically in the collenchyma cells and epidermis, the levels of the H3K4me3 and H3K9me2 were similar (Figure 3E).

The cellular details observed with this method show, in a more detailed resolution (Figure 4), that the signal of H3K4me3 and H3K9me2 inside the nucleus of the 


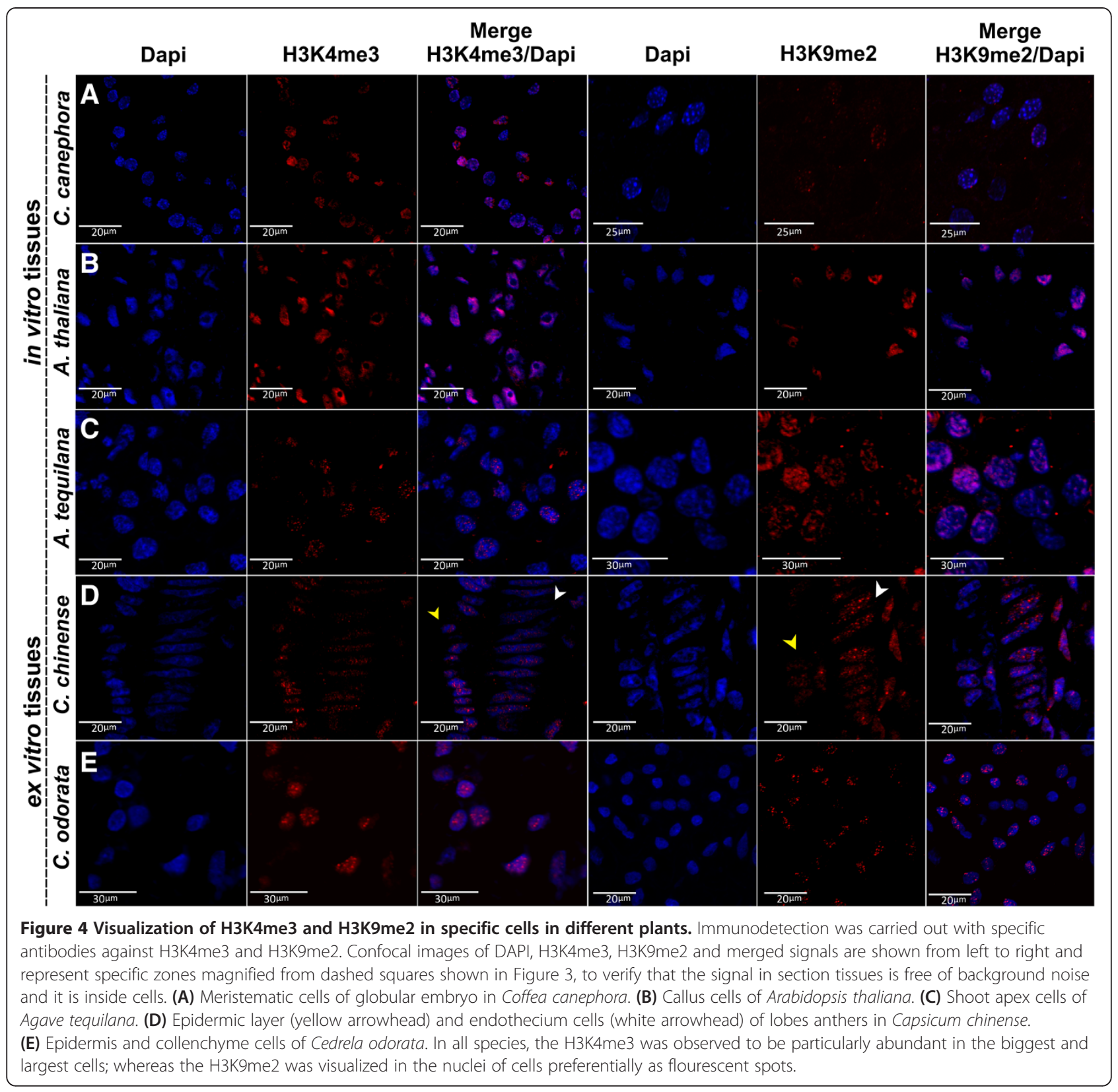

cells was free of background noise and very specific. For instance, it was found that the cells that contain the strong signal intensity of H3K4me3, which is related to transcriptional activity, was localized mainly in big nucleus, while that of $\mathrm{H} 3 \mathrm{~K} 9 \mathrm{me} 2$ was observed in the compacted nucleus, particularly restricted to heterochromatic zones (Figure 4).

In the case of the globular embryo of C. canephora, the signals of H3K4me3 in the inner cells were clearly visible, which was consistent with the low signal of H3K9me2; this probably suggests a transcriptional activity in this area (Figure 4A). This same pattern was also observed in the A. thaliana callus (Figure 4B) in the central zone of the meristematic region. On the other hand, in the meristematic cells of the A. tequilana shoot apex, a low signal of H3K4me3 was detected, while high signals of H3K9me2 were seen (Figure 4C).

In C. chinense, high levels of H3K9me2 were observed in the endothecium cells (subepidermal layer that develops fibrous wall thickenings in late anther development) but only a slight increase of H3K4me3 in the epidermis cells of the anther lobes was detected. These results could be related to the immature anther, because even the epidermal layer is transcriptionally active, but that frequently disappears before anther maturity to expose the endothecium [40]. In the case of C. odorata, 
it was possible to detect the distribution of high levels of H3K4me3 in the epidermis cells, whereas H3K9me2 occurs preferentially in the collenchyma cells of the bud (Figure 4E). These results provide information about the cell types and their possible activity during the vegetative growth of the buds.

The results presented in this work propose an efficient immunolocalization method that can be used in different plant tissues and plant growth conditions. Moreover, deposition of the epigenetic marks H3K4me3 and H3K9me2 were found in different tissues, including callus, flowers, buds, somatic embryos and vascular procambium, as well as in different conditions (in vitro and ex vitro). The differential localization of the epigenetic marks analyzed with this protocol revealed the complex spatial distribution of $\mathrm{H} 3 \mathrm{~K} 4 \mathrm{me} 3$ and $\mathrm{H} 3 \mathrm{~K} 9 \mathrm{me} 2$ that could indicate the efficient use of epigenetic mechanisms by different types of plants and tissues to regulate gene transcription during their growth and development or in response to environmental stimuli $[7,27]$.

\section{Conclusions}

Here we provide an easy, efficient and inexpensive method to carry out the immunodetection of histone methylation; it is likely to be suitable also for use with a wide spectrum of antibodies. This method has been shown to be effective with several plants of agronomical and biological importance, such as coffee, agave, chili pepper, cedar and Arabidopsis, and in controlled environmental conditions (in vitro), or in looking at plants exposed to uncontrolled conditions or that are difficult to cultivate in the laboratory, such as cedar (ex vitro). Furthermore, in this work, we have shown that antigen retrieval performed by microwave heating and the use of citrate buffer improves the immunodetection process and enhances signal detection and the quality of the immunolocalization. The spatial distribution and cellular localization of the proteins of interest can be obtained by this simple, highly reproducible and inexpensive method, which could be applied to different tissues of other plant species.

\section{Methods}

\section{Plant material}

Cedrela odorata plantlets and flowers of Capsicum chinense were collected at the greenhouse at CICY, Mérida, Yucatán, México. In vitro Agave tequilana plantlets were cultivated in Murashige \& Skoog [41] medium supplemented with $10 \mathrm{mg} / \mathrm{L}$ BAP and $0.025 \mathrm{mg} / \mathrm{L} \mathrm{2,4-D} \mathrm{and}$ cultured under photoperiod conditions (16 hours of light $/ 8$ hours of dark at $25^{\circ} \mathrm{C}$ ). Callus of Arabidopsis thaliana (ecotype Col-0) were established from immature zygotic embryos according to Mordhorst et al. [42] and cultured under the same photoperiod conditions described above. Somatic embryos of Coffea canephora were obtained according to the method described by Quiroz-Figueroa et al. [43].

\section{Reagents}

Albumin from bovine serum (BSA) was obtained from Sigma (Cat. \# A2153). Anti-histone H3 antibody, CT, pan $(\alpha-\mathrm{H} 3$; Cat. \# 07-690), anti-trimethyl-histone H3 at lysine 4 ( $\alpha-\mathrm{H} 3 \mathrm{~K} 4 \mathrm{me} 3$; Cat. \# 05-745R) and anti-dimethylhistone H3 at lysine $9(\alpha-\mathrm{H} 3 \mathrm{~K} 9 \mathrm{me} 2$; Cat. \# 07-441) were purchased from Millipore. Alexa Fluor 647 goat antirabbit IgG was obtained from Invitrogen (Cat. \# A21244). VECTASHIELD ${ }^{\circ}$ mounting medium with 4,-6-diamidino2-phenylindole (DAPI) was obtained from Vector laboratories (Cat. \# H-1200).

\section{Immunodetection protocol}

Each of the plant tissues was collected and fixed in FAA solution $\left[10 \%\right.$ formaldehyde (Fischer BioReagents ${ }^{\circ}, \mathrm{BP} 531$ ), 5\% acetic acid (Sigma, 695092), 50\% absolute ethanol (Meyer $\left.\left.{ }^{\circ}, 0390\right)\right]$ for $48-72 \mathrm{~h}$ at $4^{\circ} \mathrm{C}$, changing the FAA solution every $24 \mathrm{~h}$ and applying vacuum for $10 \mathrm{~min}$ at each change. Then, the FAA was removed and the samples were dehydrated through a gradual increase of 30, 50, $70,85 \%(2 \times 2 \mathrm{~h}$ each $)$ and $96 \%(2 \times 30 \mathrm{~min}$ each $)$ of absolute ethanol, applying vacuum for $5 \mathrm{~min}$ at each step and maintaining the samples at $4^{\circ} \mathrm{C}$. After that, the samples were incubated twice for $24 \mathrm{~h}$ each in $100 \%$ butanol (J.T. Baker, 9054-03) at room temperature.

To begin the process of embedding the tissue samples in paraffin, fresh butanol and 15-30 flakes of Paraplast Plus $^{\circ}$ (Sigma, P3683) were added to the samples and incubated overnight at room temperature at $60 \mathrm{rpm}$. Then, the samples were incubated at $60^{\circ} \mathrm{C}$, adding $30-40$ flakes of paraffin every 2-3 hours, three times. The excess butanol was removed changing the liquid paraffin every 12 hours, four times. Tissue samples were placed in the center of stainless steel base molds (Fisher Scientific, $15-182-505 \mathrm{~B})$ previously heated to $60^{\circ} \mathrm{C}$ and embedded in paraffin to fill; then, the molds were covered with the cassettes' hold tissue (Fisher Scientific, 15182702A). The cassettes were removed after $3-4 \mathrm{~h}$ at room temperature and the paraffin-embedded sample was sectioned into 4-5 $\mu \mathrm{m}$ slices using a retracting microtome $\left(\mathrm{MICROM}^{\circ}\right.$, HM 325) with low profile blades (Thermo Scientific, 1407060). The sections were placed in a $42^{\circ} \mathrm{C}$ water bath to allow the correct expansion of the tissues, and then placed on micro slides (Sigma, S8400). Tissue sections attached to the micro slides were maintained at $37^{\circ} \mathrm{C}$ at least for 2 hours. From this step, the tissue sections can be stored at $4^{\circ} \mathrm{C}$ for several months without losing tissue integrity.

Paraffin removal was accomplished as follows: dried sections were incubated at $65^{\circ} \mathrm{C}$ for $15 \mathrm{~min}$ and deparaffinized 
in slide staining jars (Fisher Scientific, 08-813E) with xylene, (Sigma, 534056) three times for $10 \mathrm{~min}$ per rinse, and ultraclear (J.T. Baker, 3905), four times every $15 \mathrm{~min}$ per rinse. The ultraclear was removed and the slides were washed twice in absolute ethanol at $100 \%$ for 2 min per rinse. Then, the tissue sections were rehydrated with a series of absolute ethanol-water combinations (96, 80, 70, 50 and $30 \%$ for $5 \mathrm{~min}$ in each step) and water twice (5 min each).

Antigen retrieval was carried out by rinsing the slides in citrate buffer $[10 \mathrm{mM}$ Sodium citrate dehydrate (Sigma, W302600) at $\mathrm{pH} 6$ adjusted with $1 \mathrm{~N}$ of $\mathrm{HCl}$, and $0.05 \%$ Tween $^{\oplus} 20$ (Sigma, P1379); this solution can be stored at $4^{\circ} \mathrm{C}$ ] and microwave-heated at high power for $4 \mathrm{~min}$. In order to prevent tissue drying during the microwave exposure, the amount of buffer needs to be enough to cover the sample. After heating, the slides were rinsed with warm water to slowly cool the temperature of the slide over 5 min and, then, the slides with the sample tissue were washed three times with PBS buffer [150 mM sodium chloride crystal (J.T. Baker, 3624-01), $10 \mathrm{mM}$ sodium phosphate dibasic (Sigma, S3264), $2 \mathrm{mM}$ potassium phosphate monobasic (Sigma, P5655) $\mathrm{pH} 7.2$ adjusted with $1 \mathrm{~N} \mathrm{NaOH}$ ] for 5 min each. The PBS buffer should be freshly prepared and filtered through a $0.45-\mu \mathrm{m}$ membrane.

Excess of PBS was carefully removed around the tissue section in the slides with absorbent paper and incubated with 20-30 $\mu$ l of blocking buffer (3\% BSA diluted in PBS buffer) avoiding bubbles. The slides were incubated in a wet chamber for 1 hour at $4^{\circ} \mathrm{C}$ [wet chamber can be prepared with a moistened Whatman paper placed on the bottom of a petri dish (Sigma, CLS3160100) and tightly sealed with paraffin film]; then, the slides were washed three times with PBS buffer for 5 min each. The excess of PBS was removed again and 20-30 $\mu$ l of the primary antibody (anti-H3, anti-H3K4me3 and anti$\mathrm{H} 3 \mathrm{~K} 9 \mathrm{me} 2)$ was added to each tissue section, and then the slides were incubated overnight at $4^{\circ} \mathrm{C}$ in a wet chamber. After the washes in PBS buffer $(3 \times 5 \mathrm{~min})$, the PBS was removed and the sections were incubated with 20-30 $\mu$ l of the fluorescently labeled secondary antibody Alexa Fluor 647 for another 3 hours in a wet chamber at room temperature. The dilution of both primary and secondary antibodies was performed in $1 \%$ BSA diluted in PBS buffer at 1:200 and 1:100, respectively. Subsequent washes in PBS buffer $(3 \times 10 \mathrm{~min})$ were carried out while protecting the slides from the light. Finally, the tissue sections in the slides were counterstained with $20 \mu$ l of Vectashield ${ }^{\circledR}$ mounting medium, with DAPI to stain the DNA, mounted with a $22 \times$ $22 \mathrm{~mm}$ cover glass (Sigma, C9802) and sealed around the perimeter with nail polish or a plastic sealant and stored in the dark at $4^{\circ} \mathrm{C}$ prior to analysis. The photographs were obtained using a confocal laser scanning microscope (Olympus, FV100 SW) and the FV10 ASW 3.1 viewer software. The H3, H3K4me3 and H3K9me2 signals were detected using an excitation wavelength at $650 \mathrm{~nm}$, and the DAPI staining signal was detected using the excitation wavelength of $405 \mathrm{~nm}$.

\section{Additional files}

Additional file 1: Immunodetection of histone $\mathrm{H} 3$ in different plant species tissues without the microwave treatment (negative control). Immunodetection against histone $\mathrm{H} 3$ avoiding the antigen retrieval from the microwaved citrate buffer in the globular embryo of Coffea canephora (A), meristematic zones in the callus of Arabidopsis thaliana (B), procambium zone of Agave tequilana $(\mathrm{C})$, anthers of Capsicum chinense (D) and bud of Cedrela odorata (E).

Additional file 2: Immunodetection of $\mathrm{H} 3 \mathrm{~K} 4 \mathrm{me} 3$ in different plant species tissues without the microwave treatment (negative control). Immunodetection against $\mathrm{H} 3 \mathrm{~K} 4 \mathrm{me} 3$ avoiding the antigen retrieval from the microwaved citrate buffer in the epidermis cells of globular embryo of Coffea canephora (A), meristematic zones in the callus of Arabidopsis thaliana (B), shoot apex of Agave tequilana (C), anthers of Capsicum chinense (D) and in the proximal cells to epidermis of Cedrela odorata (E). Dashed squares represent the close-up of the sites analyzed by immunodetection against $\mathrm{H} 3 \mathrm{~K} 4 \mathrm{me} 3$ without antigen retrieval.

Additional file 3: Immunodetection of H3K9me2 in different plant species tissues without the microwave treatment (negative control). Immunodetection against $\mathrm{H} 3 \mathrm{~K} 9 \mathrm{me} 2$ avoiding the antigen retrieval from the microwaved citrate buffer in the epidermis of globular embryo of Coffea canephora (A), meristematic zones in the callus of Arabidopsis thaliana (B), shoot apex of Agave tequilana (C), anthers of Capsicum chinense (D) and in the proximal cells to epidermis of Cedrela odorata (E). Dashed squares represent the close-up of the sites analyzed.

\section{Abbreviations}

H3: Histone H3; H3K4me3: Trimethyl-histone H3 at Lys4; H3K9me2: Dimethylhistone $\mathrm{H} 3$ at Lys9; BSA: Albumin from bovine serum; DAPI: 4,-6-diamidino-2phenylindole.

\section{Competing interests}

The authors declare that they have competing interests.

\section{Authors' contributions}

GNC conceived the protocol, collected the tissue samples, did the immunodetection and drafted the manuscript. SHC performed the histology of samples and helped with immunodetection assays. AKG performed the confocal image analysis. VMLV and CDP coordinated the project, helped with the design of the study and assisted with the drafting of the manuscript. All authors read and approved the final manuscript.

\section{Acknowledgments}

This work was supported by grants received from the National Council for Science and Technology (CONACyT; 157014 to VMLV and 178149 to CDP). GNC was supported by a scholarship (213451) from CONACyT. We thank Alma Laura Rodríguez Piña and Eduardo Castillo Castro for providing us the callus cultures of Arabidopsis thaliana and the plantlets of Agave tequilana, respectively.

\section{Author details}

${ }^{1}$ Unidad de Bioquímica y Biología Molecular de Plantas, Centro de Investigación Científica de Yucatán, Calle 43 No. 130, Col. Chuburná de Hidalgo, Mérida CP 97200, Yucatán, México. ${ }^{2}$ Unidad de Biotecnología, Centro de Investigación Científica de Yucatán, Calle 43 No. 130, Col. Chuburná de Hidalgo, Mérida CP 97200, Yucatán, México.

Received: 19 August 2013 Accepted: 2 December 2013

Published: 16 December 2013 


\section{References}

1. Vanyushin B, Ashapkin W: DNA methylation in higher plants: past, present and future. Biochim Biophys Acta 1809, 2011:360-368.

2. Kouzarides T: Chromatin modifications and their function. Cell 2007, 128:693-705.

3. Zhang K, Sridhar V, Zhu J, Kapoor A, Zhu JK: Distinctive core of histone post-translational modification patterns in Arabidopsis thaliana. Plos One 2007, 2:e1210.

4. Bannister J, Kouzarides T: Regulation of chromatin by histone modifications. Cell Res 2011, 21:381-395.

5. Li X, Wang X, He K, Ma Y, Su N, Stolc V, et al: High-resolution mapping of epigenetic modifications of the rice genome uncovers interplay between DNA methylation, and gene expression. Plant Cell 2008, 20:259-276.

6. Li H, Freeling M, Lisch D: Epigenetic reprogramming during vegetative phase change in maize. Proc Natl Acad Sci USA 2010, 107:22184-22189.

7. Zhang X, Bernatavichute YV, Cokus S, Pellegrini M, Jacobsen S: Genomewide analysis of mono-, di- or trimethylation of histone $\mathrm{H} 3$ lysine 4 in Arabidopsis thaliana. Gen Biol 2009, 10:R62

8. Stimpson K, Sullivan B: Histone H3K4 methylation keeps centromeres open for business. EMBO J 2011, 30:233-234.

9. Xu L, Zhao Z, Dong A, Taconnat L, Renou J, Steinmetz A, et al: Di- and tri- but not monomethylation on histone $\mathrm{H} 3$ lysine 36 marks active transcription of genes involved in flowering time regulation and other processes in Arabidopsis thaliana. Mol Cel Biol 2008, 28:1348-1360.

10. Sui $P$, Jin J, Mu C, Gao J, Feng H, Shen W, et al: H3K36 methylation is critical for brassinosteroid-regulated plant growth and development in rice. Plant J 2012, 70:340-347.

11. Naumann K, Fischer A, Hofmann I, Krauss V, Phalke S, Irmler K, et al: Pivotal role of AtSUVH2 in heterochromatin histone methylation and gene silencing in Arabidopsis. EMBO J 2005, 24:1418-1429.

12. Veiseth S, Rahman M, Yap K, Fischer A, Jacobsen W, Reuter G, et al: The SUVR4 histone lysine methyltransferase binds ubiquitin and converts $\mathrm{H} 3 \mathrm{~K} 9 \mathrm{me} 1$ to H3K9me3 on transposon chromatin in Arabidopsis. Plos Genet 2011, 7:e1001325.

13. Zhang X, Clarenz O, Cokus S, Bernatavichute YV, Pellegrini M, Goodrich J, et al: Whole-genome analysis of histone $\mathrm{H} 3$ lysine 27 trimethylation in Arabidopsis. Plos Biol 2007, 5:1026-1035.

14. Zheng B, Chen X: Dynamics of histone $\mathrm{H} 3$ lysine 27 trimethylation in plant development. Curr Opi Plant Biol 2011, 14:123-129.

15. Thorstensen T, Grini PE, Aelen RB: SET domain proteins in plant development. Biochim Biophys Acta 1809, 2011:407-420.

16. Saleh A, Alvarez R, Avramova Z: An efficient chromatin immunoprecipitation (Chip) protocol for studying histone modifications in Arabidopsis plants. Nat Prot 2010, 3:1018-1025.

17. Deal RB, Henikoff J, Henikoff S: Genome-wide kinetics of nucleosome turnover determined by metabolic labeling of histones. Science 2010, 328:1161-1164.

18. Perrella G, Consiglio M, Aiese R, Cremona G, Sanchez M, Barra L, et al: Histone hyperacetylation affects meiotic recombination and chromosome segregation in Arabidopsis. Plant J 2010, 62:796-806.

19. Ay N, Irmler K, Fischer A, Uhlemann R, Reuter G, Humbeck K: Epigenetic programming via histone methylation at WRKY53 controls leaf senescence in Arabidopsis thaliana. Plant J 2009, 58:333-346.

20. Santamaría E, Hasbún R, Valera J, Meijón M, Valledor L, Rodríguez J, et al: Acetylated $\mathrm{H} 4$ histone and genomic DNA methylation patterns during bud set and bud burst in Castanea sativa. J Plant Physiol 2009 166:1360-1369.

21. Meijón M, Feito I, Valledor L, Rodríguez R, Cañal MJ: Dynamics of the DNA methylation and Histone $\mathrm{H} 4$ acetylation during floral bud differentiation in azalea. BMC Plant Biol 2010, 10:10.

22. Smertenko A, Hussey P: Immunolocalization of proteins in somatic embryos. In Plant Embryogenesis. Edited by Suárez MF, Bozhkov PV. Totowa: Humana Press; 2008:157-171.

23. Yang $X$, Yuan $L$, Makaroff C: Immunolocalization protocols for visualizing meiotic proteins in Arabidopsis thaliana: Method 3. In Plant Meiosis, Methods in Molecular Biology. Edited by Pawloski W. New York: Springer +Business Media; 2013:109-118

24. Shi S, Key M, Kalra K: Antigen retrieval in formalin-fixed, paraffinembedded tissues: an enhancement method for immunohistochemical staining based on microwave oven heating of tissue sections. J Histochem Cytochem 1991, 39:741-748.

25. Jasencakova Z, Soppe W, Meister A, Turner B, Schubert I: Histone modifications in Arabidopsis-high methylation of H3 lysine 9 is dispensable for constitutive heterochromatin. Plant J 2003, 33:471-478.

26. Hou Z, Huang W: Immunohistochemical localization of IAA and ABP1 in strawberry shoot apexes during floral induction. Planta 2005, 222:678-687.

27. Yao X, Feng H, Yu Y, Dong A, Shi Y, Shen WH: SDG2-Mediated H3K4 methylation is required for proper Arabidopsis root growth and development. Plos One 2013, 8:e56537.

28. Friml J, Benkova E, Mayer U, Palme K, Muster G: Automated whole mount localization techniques for plant seedlings. Plant J 2003, 34:115-124

29. Shi S, Cote R, Taylor C: Antigen retrieval techniques: current perspectives. $\checkmark$ Histochem Cytochem 2001, 49:931-937.

30. Koltai H, Bird D: High throughput cellular localization of specific plant mRNAs by liquid-phase in situ reverse transcription-polymerase chain reaction of tissue sections. Plant Physiol 2000, 123:1203-1212.

31. Shi S, Cheng Q, Zhang P, Wang N, Zheng Y, Bai X, et al: Immunoflourescence with dual microwave of paraffin-embedded sections in the assessment of human renal biopsy specimens. Am J Clin Pathol 2013, 139:71-78.

32. Shi S, Zhang P, Cheng Q, Wu J, Cui J, Zheng Y, et al: Immunohistochemistry of deparaffinized sections using antigen retrieval with microwave combined pressure cooking versus immunoflourescence in the assessment of human renal biopsies. J Clin Pathol 2013, 66:374-380.

33. Benhamou N, Noel S, Grenier J, Asselin A: Microwave energy fixation of plant tissue: an alternative approach that provides excellent preservation of ultrastructure and antigenicity. J Elec Microsc Tech 1991, 17:81-94

34. Medina FJ, Cerdido A, Maroto M, Manzanares M, Marco R: Enhancement of the immunocytochemical detection of antigens by microwave irradiation. Benefits and limitations analyzed in isolated plant nuclei and Drosophila embryos in toto. Histochemistry 1994, 102:45-50.

35. Lería F, Marco R, Medina FJ: Structural and antigenic preservation of plant samples by microwave-enhanced fixation, using dedicated hardware, minimizing heat-related effects. Microsc Res Tech 2004, 65:86-100.

36. Sibony M, Commo F, Callard P, Gasc J: Enhancement of mRNA in situ hybridization signal by microwave heating. Lab Invest 1995, 73:586-591.

37. Nic-Can G, De la Peña C: Determination of histone methylation in mono- and dicotyledonous plants. In Plant Cell Culture Protocols, Methods in Molecular Biology. Edited by Loyola-Vargas VM, Ochoa-Alejo N. Heidelberg: Humana Press; 2012:313-324.

38. De-la-Peña C, Nic-Can G, Ojeda G, Herrera J, Lopez Torres A, Wrobel K, et al: KNOX1 is expressed and epigenetically regulated during in vitro conditions in Agave spp. BMC Plant Biol 2012, 12:203.

39. Jackson JP, Johnson L, Jasencakova Z, Zhang $X$, Burgos L, Singh $P$, et al: Dimethylation of histone $\mathrm{H} 3$ lysine 9 is a critical mark for DNA methylation and gene silencing in Arabidopsis thaliana. Chromosoma 2004, 112:308-315.

40. Borg M, Brownfield L, Twell D: Male gametophyte development: a molecular perspective. J Exp Bot 2009, 60:1465-1478.

41. Murashige T, Skoog F: A revised medium for rapid growth and bioassays with tobacco tissue cultures. Physiol Plant 1962, 15:473-497.

42. Mordhorst A, Hartog M, El Tamer M, Laux T, De Vries S: Somatic embryogenesis from Arabidopsis shoot apical meristem mutants. Planta 2002, 214:829-836.

43. Quiroz-Figueroa FR, Monforte-González M, Galaz-Avalos RM, Loyola-Vargas VM: Direct somatic embryogenesis in Coffea canephora. In Plant cell culture protocols. Edited by Loyola-Vargas VM, Vázquez-Flota FA. Totowa, New Jersey: Humana Press; 2006:111-117.

doi:10.1186/1746-4811-9-47

Cite this article as: Nic-Can et al:: An efficient immunodetection method for histone modifications in plants. Plant Methods 2013 9:47. 